\title{
PHYSICAL PROCESSES IN THE PLASMA MANTLE OF VENUS
}

\author{
K. Szegö ${ }^{1}$, V.S. Shapiro ${ }^{2}$, V.I. Shevchenko ${ }^{2}$, R.Z. Sagdeev ${ }^{3}$, \\ W.T. Kasprzak ${ }^{4}$, and A.F. Nagy
}

\begin{abstract}
The Phobos-2 observations established the presence of a turbulent region on the dayside, called the mantle, just outside the magnetic barrier at Mars. This mantle is the interaction region of cold Martian ions and the shocked solar wind. This work presents the results of a study, which andyzed data from ten Pioneer Venus orbits, in order to see whether similar wave particle interaction processes also exist in the corresponding region around Venus. The first conclusion is that the apparent physical processes in the mantle are indeed similar around Venus and Mars. The planetary thermal $\mathrm{O}^{+}$ions outside the ionopause interact with the shocked solar wind and excite electrostatic waves close to the lower hybrid frequency. These wave propagate inwards, heating first the electron and deeper down in the ionosphere the thermal ion population. The observed superthermal ions are believed to be the product of this wave particle interaction process. Our second conclusion is that the wave energy transferred to the thermal electrons is of the right magnitude $\left(\sim 4 \times 10^{9} \mathrm{eV} \mathrm{cm}^{-2} \mathrm{~s}^{-1}\right)$ to provide the supplemental heat source necessary to reconcile observed and calculated electron temperatures in the ionosphere.
\end{abstract}

\section{Introduction}

The exploration of the magnetosphere of Mars by the field and particle instruments carried onboard the Soviet PHOBOS2 spacecraft, revealed the existence of a turbulent transition region just outside of the dayside magnetic barrier. In this region the wave signature, ion, and electron population were different both from those in the shocked solar wind and those found in regions closer to the planet. The physical processes were dominated by the turbulent interaction of cold planetary ions and the shocked solar wind [Sagdeev et al., 1990]. The question immediately arose whether the situation is similar around Venus [Nagy et al., 1990] and around other non magnetic solar system bodies [Szego et al., 1991]. This paper is dedicated to a more detailed study of this region in question around Venus.

In earlier investigations the existence of a transition region called the mantle - between the ionosphere and the ionosheath/magnetosheath was established by the low energy $(\sim 5-60 \mathrm{eV})$ electron observations of the Retarding Potential Analyzer (RPA) instrument on the Pioneer Venus Orbiter (PVO) [Spenner et al., 1980]. Suprathermal ion clouds were also found to be present, in this mantle region, by the Orbiter Ion Mass Spectrometer (OMMS) [Taylor et al., 1981]. Though this instrument was designed to measure thermal ions, it could also establish the presence of these energized planetary ions in the range of 10 to $90 \mathrm{eV}$, without being able to specify the exact composition or flux values. The $100 \mathrm{~Hz}$ channel of the Orbiter Electric Field Detector (OEFD) also indicated that

\footnotetext{
I Central Research Institute for Physics

2 Space Research Institute

3 University of Maryland

4 Goddard Space Flight Center

separtment of Atmospheric, Oceanic and Space Sciences, University of Michigan
}

Copyright 1991 by the American Geophysical Union.

Paper number 91GL02086

0094-8534/91/91GL-02086\$03.00 waves, close to this frequency range, are also a permanent feature of this region [Scarf et al., 1980]. The polarizations of these waves were not measured; however Scarf et al. [1980] identified these waves as whistlers by analogy with observations in the Earth magnetosphere. On the other hand Elphic et al. [1981], concluded that these waves might be electrostatic ion acoustic waves or lower hybrid waves generated at the ionopause. Taylor et al. [1981] suggested that these waves are generated locally by planetary ions interacting with the flowing ionosheath plasma. They also pointed out correlations between the intensity of the $100 \mathrm{~Hz}$ waves and the presence of suprathermal ions. These wave observations were recently reviewed by Strangeway [1991]. While the existence of the mantle was recognized by Spenner et al., [1980], it was not studied any further.

It has been known since the Pioneer Venus observations became available, that in order to account for the observed electron and ion temperatures in the ionosphere heat source(s) beyond solar EUV radiation are required [cf. Nagy et al., 1983]. Absorption of "whistler waves" by the ionospheric plasma was considered a viable energy source, but as they propagate parallel to the magnetic field lines, for effective energy transfer other type of waves are necessary. A similar energy source is also needed to account for the observed ionospheric temperatures at Mars [Chen et al., 1978]. In our model, an appropriate mechanism for the "missing" energy source is provided.

For the study presented in this paper ten PVO orbits were selected; these orbits were chosen such that the PVO Orbital Neutral Mass Spectrometer (ONMS) could, in principle, provide information on suprathermal ions above about $40 \mathrm{eV}$ to several hundred $\mathrm{eV}$. On some of the orbits the OMMS also provided information on these suprathermal ions. The results of the data analysis are presented next; this is followed by our interpretation of the observations.

\section{Data Analysis}

The ten PVO orbits were selected in such a way that the dayside magnetosphere could be sampled as a function of the solar zenith angle. The orbits used are given in Table 1. Orbits $195,207,211,414$ and 428 did not cross the bowshock on the dayside. For the other orbits the unperturbed

TABLE 1.

\begin{tabular}{lrlccc}
\hline Orbit No. & Angle & SW vel. & sW press & In & Out \\
\hline 148 & -62 & 448 & 2.6 & 1051 & 1165 \\
173 & -24 & 309 & 2.6 & 520 & 405 \\
400 & -20 & 308 & 1.6 & 1208 & 943 \\
414 & 2 & & & 394 & 742 \\
195 & 12 & & & 520 & 382 \\
428 & 25 & & & 850 & 577 \\
207 & 31 & & & 364 & 601 \\
211 & 37 & & & 452 & 604 \\
442 & 47 & 301 & 5.6 & 695 & 581 \\
226 & 62 & 358 & 2.6 & 749 & 1020 \\
\hline
\end{tabular}

The pertinent data of the 10 orbits. Orbit No. is the PVO orbit number; the angle is the solar zenith angle of the periapsis; SW vel. is the velocity of the unperturbed solar wind in $\mathrm{km} / \mathrm{s}$, SW press. is the dynamical pressure in $10^{-8} \mathrm{dyn} / \mathrm{cm}^{2}$; In and Out give the altitude of the magnetic barrier (in $\mathbf{k m}$ ) during the inbound and outbound portions of the orbit. 
solar wind dynamic pressure and velocity are also listed in Table 1 as measured by the OPA (J. Mihalov, private communication). Shocked solar wind data between the bowshock and the dayside ionosphere were not accessible to us.

Magnetic field and wave data were available for all ten orbits, and with the exception of orbits 400 and 414 so were the densities of the thermal $\mathrm{O}^{+}$ions (OIMS data), from the files of the publicly retrievable PVO data system. As can be seen from the data, (illustrative examples for orbit 173 are
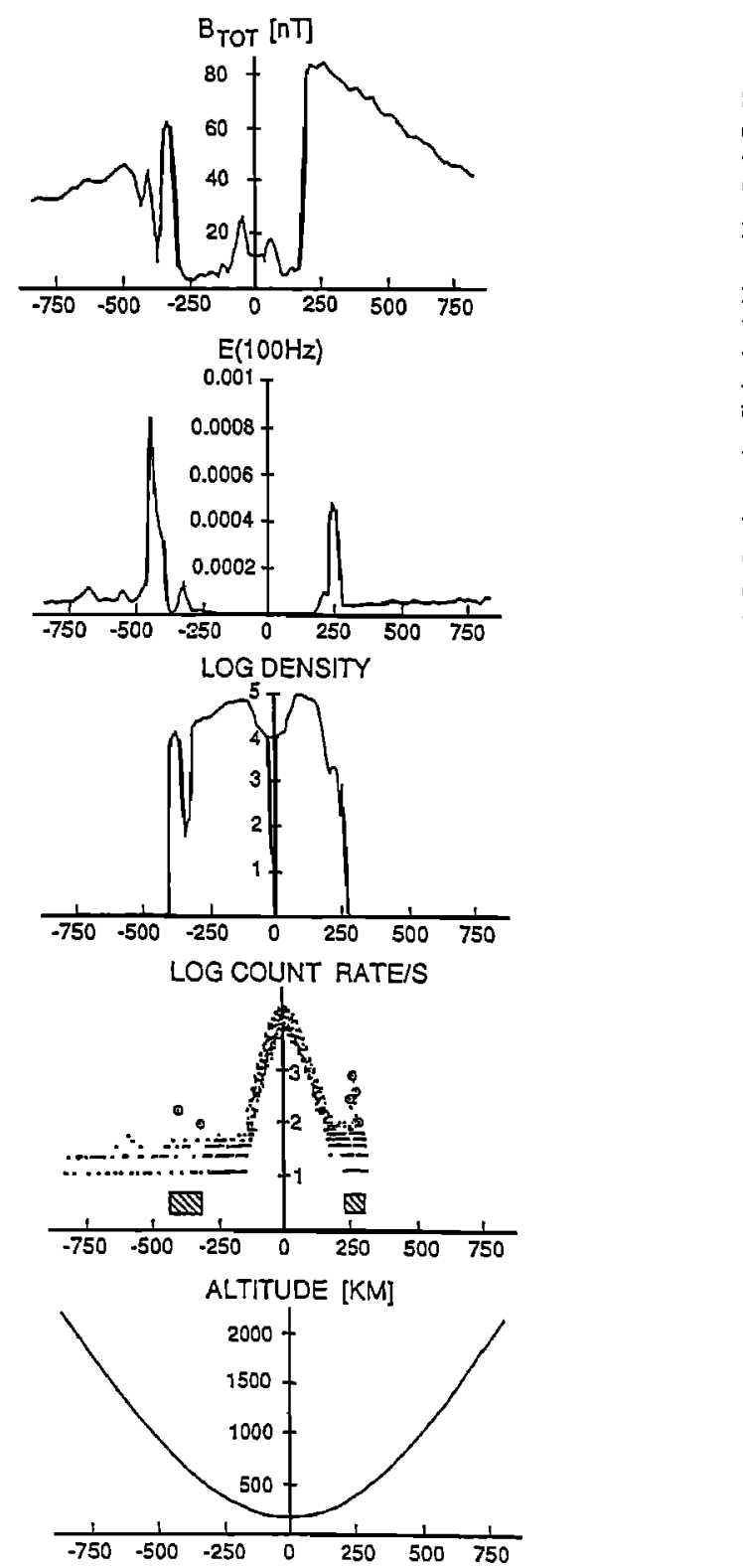

Fig. 1. From top to bottom the data shown from Orbit 173 are:

- the variation of the total magnetic field in $\mathrm{nT}$,

- the wave fields in the $100 \mathrm{~Hz}$ channel of OEFD in $\mathrm{V} / \mathrm{m} / \mathrm{VHz}$,

- the logarithm of the cold $0^{+}$ion density in ion $/ \mathrm{cm}^{3}$ from OIMS,

- the logarithm of the count rate per second in channel 16 of ONMS. The dots denote neutrals, dots in circles are suprathermal ion events. The time periods during which the OIMS observed suprathermal ion events are also indicated here by the crosshatched areas,

- and the altitude of the orbiter.

On the horizontal axes the time indicated is in seconds relative to periapsis. given in Figure 1), the peak value of the $100 \mathrm{~Hz}$ signal appeared always at a somewhat higher altitude than the peak value of the the magnetic field (the narrow region around this magnetic field enhancement is called the magnetic barrier); also the tail of the cold $0^{+}$distribution always extended to the altitude where the $100 \mathrm{~Hz}$ signals were observed.

The ONMS was designed to measure neutrals in a cold ambient ion environment. To avoid cold ions, the device had a repeller grid, adjusted to $36 \mathrm{~V}$. However, ions with a directed energy greater than $36 \mathrm{eV}$ do reach the detector [Kasprzak et al., 1982]. So if the ambient neutral density was low enough (i.e., the spacecraft was not at low enough altitude), sufficiently large enough suprathermal ion fluxes could be detected (cf. Figure 1). If the density of suprathermal ions was too low, the signal due to them disappeared in the 'background', i.e., it could not be distinguished from the neutral count rate. The ONMS data were processed for orbits 173,195 and 414, some of the measured suprathermal ion flux values are given in Table 2. The flux was derived from the instrument count rate by a least square fitting procedure with an average flux value being generated approximately once per 12 second spacecraft spin period [Kasprzak et al., 1987]. The location of the peak fluxes (count rates) indicates that these particles are unlikely to have originated near the bow shock. Data for the other orbits are yet unprocessed. There are visual signatures of the presence of superthermal ions on orbits 207,211 and 442 , no unambiguous signals were observed along the other orbits. The fluxes of the ions are of the order of $10^{6}-10^{7} \mathrm{~cm}^{-2} \mathrm{~s}^{-1}$ and they are observed in approximately the same region where the wave signals in the $100 \mathrm{~Hz}$ channel are present.

TABLE 2.

\begin{tabular}{lclllll}
\hline Orbit No. & \multicolumn{2}{c}{173} & \multicolumn{2}{c}{195} & \multicolumn{2}{c}{414} \\
& alt & flux & alt & flux & alt & flux \\
\hline & & & 428 & $8.6 \mathrm{E6}$ & 1431 & $1.1 \mathrm{E6}$ \\
& 406 & $1.5 \mathrm{E6}$ & 405 & $1.9 \mathrm{E} 7$ & 1385 & $2.7 \mathrm{E} 6$ \\
& 387 & $3.1 \mathrm{E} 6$ & 385 & $1.0 \mathrm{E} 7$ & 1343 & $5.6 \mathrm{E} 6$ \\
& & & 373 & $2.8 \mathrm{E} 6$ & 365 & $6.9 \mathrm{E} 6$ \\
\hline
\end{tabular}

ONMS fluxes in cm $-2 \mathrm{~s}-1$ for three PVO orbits for different altitudes given in $\mathrm{km}$.

The OMS, a Bennett ion mass spectrometer was designed to analyze cold ion composition during typical ionospheric conditions [Taylor et al., 1980]. It can provide no quantitative information on suprathermal ions, although in a qualitative manner it does indicate the presence of such ions. The upper limit of the instrument response is about $100 \mathrm{eV}$. Out of the 10 orbits we investigated, there are OIMS data for orbits 173 , 195,207 and 428 which were provided to us by Dr. J. M. Grebowsky. For the first three of these orbits the "suprathermal ion events" are well correlated in altitude with the ONMS data; this is illustrated in Figure 1 for the case of orbit 173. On other orbits, the OMMS apparently detected the presence of energetic ions already at altitudes higher than did the ONMS. Differences are probably only due to the different sensitivity of the instruments. For orbit $\mathbf{4 2 8}$ there are OMMS "superthermal signals", but the number of events are sparse.

We also investigated whether the electron signatures of the mantle (a tail in the differential energy spectra, as at Mars) can be identified in these orbits. The RPA measured the integral electron energy spectra quite frequently, and these data were provided to us by Dr. W. C. Knudsen. It is evident even from the integral spectra that the electron distribution in the mantle region, i.e., close to the ionopause is different from that observed in the sheath [W. C. Knudsen, private communication].

In summary, we find that in 6 cases out of 10 there is a well defined region above the dayside ionosphere, the mantle, 
where suprathermal ions, $100 \mathrm{~Hz}$ waves and unique electron signatures are seen, as was discussed in the Introduction. The location of the magnetic barrier for the 10 orbits is given in Table 1 . The two orbits, 148 and 226 , during which no visual evidence of suprathermal ions was found, crossed the magnetic barrier at a much higher altitude than the other orbits considered in this study. At these high altitudes, in excess of $1000 \mathrm{~km}$, the planetary ion density is very low, which may affect the processes responsible for the creation of these suprathermal ions, as will be discussed in the next section; i.e., the effectiveness of ion energization is dependent on the solar zenith angle. Orbit 428 does not lend itself to any easy conclusion and for orbit 400 important ion data are missing.

\section{The Physical Processes in the Mantle}

We consider the mantle as a special region where the shocked solar wind encounters cold ions of planetary origin, and the interaction of these two major ion populations regulates the most important phenomena seen there. This concept is basically not new [e.g., Elphic et al., 1981]. The new elements are the theoretical calculations, the results of which are in good agreement with the observations and the role of the excited waves as a heat source for electrons and ions in the ionosphere. The indicated similarity of the physical processes between Venus and Mars is also novel. As was shown in the previous section, the cold $\mathrm{O}^{+}$population around Venus has a tail extending beyond the ionopause with densities of the order of $10-100 \mathrm{~cm}^{-3}$, a density comparable to the shocked solar wind density in that region, which is about 50 $\mathrm{cm}^{-3}$. No information on the shocked solar wind velocity is available for these orbits in the region under study; we assume a reasonable velocity value somewhat below $100 \mathrm{~km} \mathrm{~s}^{-1}$.

The interaction of the cold planetary ions and shocked solar wind protons excite electrostatic waves via the beam particle interaction process. The oscillations are excited by resonant ions with the resonance conditions $\omega=\mathrm{ku}$ where $u$ is the shocked solar wind velocity. The dispersion relation of this process, given below, was derived in Shapiro and Shevchenko [1988]:

$$
\begin{gathered}
\frac{\omega_{\mathrm{pe}}^{2}}{\omega_{\mathrm{He}}^{2}}\left(1+\frac{\omega_{\mathrm{pe}}^{2}}{\mathrm{k}^{2} \mathrm{c}^{2}}\right)-\frac{\omega_{\mathrm{pc}}^{2}}{\omega^{2}} \frac{\mathbf{k}_{\mathrm{pur}}^{2}}{\mathbf{k}^{2}}\left(1+\frac{\omega_{\mathrm{pe}}^{2}}{\mathrm{k}^{2} \mathrm{c}^{2}}\right)^{-1} \\
-\frac{\omega_{\mathrm{pi}}^{2}}{(\omega+\mathrm{ku})^{2}}=\mathrm{D}(\omega, \mathrm{k})=0
\end{gathered}
$$

where $\omega_{\mathrm{He}}$ and $\omega_{\mathrm{pe}}$ are the electron gyro and plasma frequencies respectively, $\omega_{\mathrm{pi}}$ is the gyrofrequency of planetary $\mathrm{O}^{+}$ions, $\mathrm{k}_{\mathrm{par}}$ is the component of the wave vector parallel to the magnetic field. The solution of the dispersion relation is:

$\omega=\omega_{\mathrm{He}} \mathrm{kk}_{\mathrm{par}} /\left(\mathrm{k}^{2}+\omega_{\mathrm{pe}}^{2} / \mathrm{c}^{2}\right) ; \gamma=\left(\mathrm{n}_{\mathrm{i}} \mathrm{m}_{\mathrm{p}} \omega_{\mathrm{LH}}^{2} \omega / \mathrm{n}_{\mathrm{p}} \mathrm{m}_{\mathrm{i}}\right)^{1 / 3}$

where $n_{i}, n_{p}, m_{i}$, and $m_{p}$ are the densities and masses of the planetary $O^{+}$ions and solar wind protons, respectively and $\gamma$ is the growth rate of the waves. The frequency of these waves is in the vicinity of the lower-hybrid frequency (this being about $0.65 \mathrm{~B}[\mathrm{nT}] \sim 40 \mathrm{~Hz}$ in the mantle in the plasma frame), but may exceed it depending on the value of $k_{p a r} / k$; and falls in the $30 \%$ fractional bandwidth [Scarf et al., 1980b] of the OEFD $100 \mathrm{~Hz}$ channel. The numerical value of the instability shows, that the distance necessary for the instability to develop is about 10usw $/ \gamma \sim 20 \mathrm{~km}$. The inclination of the shocked solar wind velocity to the magnetic field is less than $\pi / 2$, so the ions, having velocities of $-u$ in the solar wind reference frame, excite waves with negative $\mathrm{k}_{\mathrm{par}}$. The excited waves cannot be pure whistler waves, because $\mathrm{k}>\omega / \mathrm{u} \sim 6 \times 10.5 \sim \omega_{\mathrm{pe}} / \mathrm{c}$. For pure whistler waves $\mathrm{k}<\omega_{\mathrm{pe}} / \mathrm{c}$ should hold.

We solved the dispersion relation above for $k$, assuming that
TABLE 3.

\begin{tabular}{ccc}
\hline dist $[\mathrm{km}]$ & $\omega / \mathrm{k}[\mathrm{km} / \mathrm{s}]$ & $\omega / \mathrm{k}_{\operatorname{mar}}[\mathrm{km} / \mathrm{s}]$ \\
\hline 0 & 100 & 1000 \\
22 & 86 & 800 \\
63 & 67 & 563 \\
63 & 27 & 181 \\
90 & -0 & -0 \\
\hline
\end{tabular}

Change of phase velocity as a function of distance from the excitation region.

the $\{B, u\}$ plane is either parallel or perpendicular to the local normal of the ionopause, using measured values along the orbits in question, and the resonance condition along the solar wind flow direction. The calculation shows that the waves are propagating radially inwards towards the ionosphere with decreasing phase velocity. A characteristic change for the phase velocity, $\omega / \mathbf{k}$, and the parallel phase velocity, $\omega / \mathrm{k}_{\mathrm{par}}$, is given in Table 3 as a function of the distance from the excitation region.

As a result of the decreasing phase velocity the waves can heat both the ionospheric electrons and ions but at different altitude. The phase velocity of the waves, as they propagate inward, first comes into resonance with ionospheric electrons, satisfying the resonance condition $\omega=k_{p a r} v_{e}$, where $v_{e}$ is the electron velocity. The bulk temperature of the ionospheric thermal electrons is between 1,000 and $10,000^{\circ} \mathrm{K}$, with an extended tail up to about $8 \mathrm{eV}$ [Brace and Kliore, 1991]. This means that the waves will resonate first with the tail and then deeper in the ionosphere with the thermal component of the electron distribution function (the thermal velocity of a $1 \mathrm{eV}$ electron is $\sim 400 \mathrm{~km} \mathrm{~s}^{-1}$ ). The waves heat and accelerate the electrons along the field lines. The electrons are magnetized, therefore they are confined to remain inside the ionosphere and they transfer energy, via collisions, to the bulk, thermal electron population. A very similar wave particle interaction process was proposed to explain the tail of the observed electron energy distribution in the mantle/planetosphere region of Mars [Sagdeev et al. 1990].

As the waves propagate further into the ionosphere, their decreasing phase velocity comes into resonance with the velocity of the planetary ions, $\omega / \mathrm{k}-v_{i}$. The ions are 2 to 3 times cooler than the electrons and also heavier; their thermal velocity is of the order of $1 \mathrm{~km} / \mathrm{s}$, as the RPA data [Brace and Kliore, 1991] show. The resonance region, accordingly, is about 80 to $100 \mathrm{~km}$ further inward from the wave excitation region. The waves heat and accelerate the ions. The ions are to all practical purposes not magnetized, therefore they are accelerated along the electric fields of the waves out of the ionosphere, creating a suprathermal ion population outside the magnetosphere. The waves transfer momentum to the ions, and they also pick up momentum from the shocked solar wind outside the ionopause. When the ions reach the region where they were detected by the ONMS, we may assume that their velocity is getting close to that of the shocked solar wind. (It is important to note that the geometry of the ONMS sensor puts a severe limit on the detectable ion velocity, namely the velocity component perpendicular to the spacecraft spin axis cannot be higher than $22 \mathrm{~km} \mathrm{~s}^{-1}$ ). Hence, from the fluxes given in Table 2 , we may estimate the particle density to be about $0.3-3$ $\mathrm{cm}^{-3}$. To assess the energy density transferred from the solar wind to the suprathermal ions and electrons via the wave particle interaction we could apply the quasilinear diffusion equation describing the energization process. This will be discussed in detail in a paper in preparation. Our current estimate is that there is an approximate equipartition in the energy transfer to the ions and electrons. It is worth noting that the ion energization cannot be attributed to the $E \times B$ pickup mechanism, because the distance required for acceleration by that mechanism is larger than the region in question. 
The energy flux $F$ of the waves can be calculated from the dispersion relation as:

$$
\begin{gathered}
\mathrm{F}=\omega \frac{\partial \mathrm{D}}{\partial \omega}\left(\mathrm{E}^{2} / 8 \pi\right) \frac{\mathrm{d} \omega}{\mathrm{dk}} \sim 1.5 \cdot 10^{10} \\
\left\{\frac{\mathrm{n}_{\mathrm{e}}}{1000} \cdot\left(\frac{50 \mathrm{nT}}{\mathrm{B}[\mathrm{nT}]}\right)\left(\frac{\mathrm{u}[\mathrm{km} / \mathrm{s}]}{100[\mathrm{~km} / \mathrm{s}]}\right) \mathrm{E}_{\mathrm{f}}^{2}[\mathrm{mV} / \mathrm{m} \sqrt{\mathrm{Hz}}]\right\} \mathrm{eV} / \mathrm{cm}^{2} / \mathrm{s}
\end{gathered}
$$

Considering a typical wave field amplitude of $E_{f}-0.5 \mathrm{mV} \mathrm{m}^{-1}$ $\sqrt{\mathrm{Hz}}$, the energy flux corresponds to $4 \times 10^{9} \mathrm{eV} \mathrm{cm}^{-2} \mathrm{~s}^{-1}$. This energy flux is of the right order of magnitude to reconcile measured and calculated electron temperatures; for example, Nagy et al. [1991], found that a downward electron heat flux of $5 \times 10^{9} \mathrm{eV} \mathrm{cm}^{-2} \mathrm{~s}^{-1}$ was necessary to arrive at temperatures consistent with the observed values in their two-dimensional model.

As it was mentioned before, there were cases when suprathermal ions were not detected. We attribute this to the fact that on those special orbits the ionopause intersections were at very high altitudes and accordingly the thermal $\mathrm{O}^{+}$ion density was low. Under these circumstances it could not be expected that the energetic ions would show up in the ONMS data.

\section{Discussion}

In this paper we suggest a simple mechanism to describe the physical processes operating in the mantle region above the dayside ionosphere of Venus (above the magnetic barrier), based on the interaction of the shocked solar wind with cold planetary ions. This mechanism provides explanation for most of the observed features in this region: 1) the excitation of waves detected in the $100 \mathrm{~Hz}$ channel of OEFD, 2) the presence of suprathermal ions close to the ionopause boundary, and 3) clues to the missing heating mechanism for the Venus ionosphere. The conditions are somewhat different in the plasma environment of Mars than those at Venus (e.g., the possible presence of a small intrinsic magnetic field), nevertheless the wave particle interaction process outlined here for the mantle region of Venus is new and believed to be very similar to that at Mars. We assume that the mechanism we suggested plays an important role in the appropriate region of all non-magnetic solar system bodies. Our model also suggests a new mechanism for the heating of the ionosphere, and may provide the source mechanism for the electron fluxes assumed by Gan et al. [1990].

Acknowledgments. The authors are indebted to Drs. W. C. Knudsen and J. M. Grebowsky for providing unpublished ORPA and OIMS data, respectively; and for useful consultations. We also appreciate the helpful comments of two anonymous referees. The work carried out at the University of Michigan was supported by NASA Grant NAG2-491.

\section{References}

Brace, L. H., and A. J. Kliore, The structure of the Venus ionosphere, Space Sci. Rev, 55, 81-164, 1991.

Chen, R. H., T. E. Cravens, and A. F. Nagy, The Martian ionosphere in light of the Viking observations, J. Geophvs. Res., 83, 3871, 1978.

Elphic, R. C., C. T. Russell, J. G. Luhmann, F. L. Scarf, and L.H. Brace, The Venus ionopause current sheet: Thickness, length scale and controlling factors, I Geophys. Res., 86, 11430, 1981.
Gan L., T. E. Cravens and M. Horanyi, Electrons in the ionopause boundary layer of Venus, L. Geophys. Res., 95, $19023,1990$.

Kasprzak, W. T., H. A. Taylor. L. H. Brace and H. B. Niemann, Observations of energetic ions near the Venus ionopause, Planet. Space, Sci. 30, 1107-1115, 1982.

Kasprzak, W. T., H. B. Niemann and P. Mahaffy, Observations of energetic ions on the nightside of Venus, I Geophys. Res. 92, 291-298, 1987.

Nagy, A. F., T. E. Cravens, and T. I. Gombosi, Basic theory and model calculations of the Venus ionosphere, in Venus. edited by D.M. Hunten, L. Colin, T.M Donahue and V.I. Moroz, pp. 841, The University of Arizona Press, 1983.

Nagy, A. F., T. I. Gombosi, K. Szego, R. Z. Sagdeev, V. D. Shapiro, and V. I. Shevchenko, Venus mantle - Mars planetosphere: What are the similarities and differences?, Geophys. Res. Lett., 17, 865, 1990.

Nagy, A. F., A. Korosmezey, J. Kim and T. I. Gombosi, A two dimensional, shock capturing hydrodynamic model of the Venus ionosphere, Geophys.Res.Lett., 18, 801, 1991.

Sagdeev, R. Z., V. D. Shapiro, V. I. Shevchenko, A. Zacharov, P. Kiraly, K. Szego, and A.F. Nagy, Wave activity in the neighborhood of the bow shock of Mars, Geophys. Res. Lett. 17, 893, 1990.

Scarf, F. L., W. W. L. Taylor, C. T. Russell, and R. C. Elphic, Pioneer Venus plasma wave observations: The solar wind Venus interaction, I. Geophys. Res., 85, 7599, 1980a.

Scarf, F. L., W. W. L. Taylor, and P. F. Virobik, The Poineer Venus Orbiter plasma wave investigation, IEEE Trans. Geosci., GE-18, 1, 36,1980b.

Shapiro, V. D. and Shevchenko, V. I., Astrophysical plasma turbulences, Astrophys and Space Sci.Rev, 6, 427, 1988.

Spenner, K., W. C. Knudsen, K. L. Miller, V. Novak, C. T. Russell, and R.C. Elphic, Observation of the Venus mantle, the boundary region between solar wind and ionosphere, J. Geophys. Res., 85, 7655, 1980.

Strangeway, R. J., Plasma waves at Venus, Planet. Space Sci, in press, 1991.

Szego, K., et al., On the dayside mantle region around those nonmagnetic solar system bodies which have ionosphere, Adv. Space Res, in press, 1991.

Taylor, H. A., H. C. Brinton, T. C. G. Wagner, S. J. Bauer, R. E. Hartle, B. H. Blackwell, G. R. Cordier, P. A. Cloutier and R. E. Daniell, Bennett ion mass spectrometer on the Pioneer Venus bus and orbiter, IEEE Trans. Geosci. Rem. Sens., GE-18, 44, 1980.

Taylor, H. A., et al., Dynamic variations observed in thermal and superthermal ion distribution in the dayside ionosphere of Venus,Ady. Space Res., 1, 1247, 1981.

1K. Szegö, Central Research Institute for Physics, Budapest, Hungary.

2 V. D. Shapiro and V. I. Shevchenko, Space Research Institute, Moscow, USSR.

${ }_{3}$. Z. Sagdeev, University of Maryland, College Park, MD.

${ }^{4}$ W. T. Kasprzak, Goddard Space Flight Center, Greenbelt, MD.

${ }^{5}$ A. F. Nagy, Department of Atmospheric, Oceanic and Space Sciences, University of Michigan, Ann Arbor, MI.

(Received April 8, 1991;

Revised July 24, 1991;

Accepted August 5, 1991.) 